\title{
Canonical Quantization Inside the Schwarzschild Black Hole
}

\author{
U. A. Yajnik* and K. Narayan ${ }^{\dagger}$ \\ Physics Department, Indian Institute of Technology Bombay, \\ Mumbai 400076 \\ gr-qc/9706021
}

\begin{abstract}
We propose a scheme for quantizing a scalar field over the Schwarzschild manifold including the interior of the horizon. On the exterior, the timelike Killing vector and on the horizon the isometry corresponding to restricted Lorentz boosts can be used to enforce the spectral condition. For the interior we appeal to CPT invariance to construct an explicitly positive definite operator which allows identification of positive and negative frequencies. This operator is the translation operator corresponding to the inexorable propagation to smaller radii as expected from the classical metric. We also propose an expression for the propagator in the interior and express it as a mode sum. The field theory thus obtained is meaningful for small curvatures far from the classical singularity.
\end{abstract}

To appear in Classical and Quantum Gravity

*yajnik@niharika.phy.iitb.ernet.in

†narayan@tristan.tn.cornell.edu (after September 1 1997) 


\section{Introduction}

We propose a quantization of a scalar field inside the Schwarzschild black hole. We adopt the conventional canonical quantization approach and the well known classical fact that in the Schwarzschild interior test particles move inexorably inward. It thus seems reasonable to assume that in the quantum theory we take the radial momentum operator to be positive definite in the direction of decreasing $r$ co-ordinate. However, the inclusion of out-going modes with positive outward radial wave-number $k_{\omega}$ would also be required by completeness. These then have to be interpreted as in-going modes with positive inward wave-number but of opposite charge. This generalises the CPT theorem as usually formulated in Minkowski space. There is a well known lucid explanation for the existence of antiparticles in Minkowski space due to Feynman; an interesting thought experiment was given by Weinberg [- [- Here we obtain a generalization to the black hole interior.

From the axiomatic point of view the main issue is the spectrum condition, viz., the existence of a positive definite operator whose spectrum would guarantee the existence of a ground state. For the case of curved spacetime, Haag et al [2] have formulated this as the principle of local stability of the Wightman functions. It requires that the support of the two-point function be restricted to the forward, i.e. here, the radially inward lightcone. We show that this principle holds for our quantization.

Candelas and Jensen [3] extended the Feynman Green Function with Hartle-Hawking boundary conditions to the interior of the Schwarzschild black hole. This Green Function obeys a periodic boundary condition in the time co-ordinate, and thus assumes a KuboMartin-Schwinger type state as the state of lowest energy. This is appropriate to the presence of an asymptotic heat bath. The boundary conditions that our prescription entails are similar to those of Boulware [何 who has determined the vacuum state for the global Schwarzschild manifold. We provide an a priori motivation for the vacuum state strictly in the interior and construct a causal propagator. Our results agree with those of Boulware in the interior. However, our prescription leaves open the possibility of matching with different quantizations in the exterior.

This paper is organised as follows. In section 2 we begin with quantization conditions and the re-interpretation of the modes, in section 3 we introduce the radial momentum operator. In section 4 we construct the causal propagator. Section 5 contains discussion and outlook.

\section{Quantization Inside the Schwarzschild Black Hole}

The Schwarzschild spacetime is described by the spherically symmetric line element

$$
d s^{2}=\left(1-\frac{2 m}{r}\right) d t^{2}-\frac{d r^{2}}{1-\frac{2 m}{r}}-r^{2} d \theta^{2}-r^{2} \sin ^{2} \theta d \phi^{2}
$$


in the $\{t, r, \theta, \phi\}$ coordinate system. These coordinates permit separation of variables and are suitable for finding the mode functions. We shall also need to use the so called tortoise co-ordinate[5] $r^{*}=r+2 m \ln |r / 2 m-1|$ in which the metric is

$$
d s^{2}=\left(1-\frac{2 m}{r}\right)\left(d t^{2}-d r^{* 2}\right)-r^{2} d \theta^{2}-r^{2} \sin ^{2} \theta d \phi^{2}
$$

In this form the $t-r$ part is conformal to $1+1$ Minkowski space. For $r<2 m, r^{*}$ ranges from 0 to $-\infty$ as a monotonically decreasing function of $r$. The spacetime has the Killing symmetries corresponding to time translation invariance $(t \rightarrow t+\tau)$ and rotational invariance. It has an event horizon at $r=r_{H}=2 \mathrm{~m}$. The Killing vector $\partial / \partial t$ is timelike in the exterior, null on the horizon and spacelike in the interior. The vector $\partial / \partial r$ is spacelike in the exterior and timelike in the interior. Thus, inside the black hole, the roles of time and space are reversed. Furthermore, any particle inside the black hole is inexorably drawn to the singularity. In other words, the future lightcone of a particle in the interior does not cross the horizon and necessarily terminates at the singularity. This is what motivates our prescription for quantization. For simplicity we consider a charged massless minimally coupled scalar field. The scheme however can be extended to any realistic field.

Accordingly, defining the canonically conjugate momentum

$$
\Pi_{r}=\frac{\partial L}{\partial\left(\partial_{r^{*}} \phi\right)}=\partial_{r^{*}} \phi^{\dagger}
$$

we impose the radial quantization conditions

$$
\begin{gathered}
{\left[\phi(r, t, \Omega), \frac{\partial \phi^{\dagger}}{\partial r^{*}}\left(r, t^{\prime}, \Omega^{\prime}\right)\right]=i \delta_{\Sigma}\left(t, \Omega ; t^{\prime}, \Omega^{\prime}\right)=i[-g(\Sigma)]^{-1 / 2} \delta\left(t-t^{\prime}\right) \delta\left(\Omega-\Omega^{\prime}\right)} \\
{\left[\phi(r, t, \Omega), \phi^{\dagger}\left(r, t^{\prime}, \Omega^{\prime}\right)\right]=0=\left[\frac{\partial}{\partial r^{*}} \phi(r, t, \Omega), \frac{\partial}{\partial r^{*}} \phi^{\dagger}\left(r, t^{\prime}, \Omega^{\prime}\right)\right]}
\end{gathered}
$$

These are "equal $r$ commutation relations" for the field. Quantization is performed on constant- $r$ hypersurfaces near the horizon in the interior (denoted by $\Sigma$ ), which are Cauchy surfaces in the black hole interior. Consider the local stability requirement of [2]. Paraphrased for the present case, it requires that the 2-point function $W^{(2)}\left(z_{1}, z_{2}\right)$ has support on the forward, i.e., radially inward light cone

$$
p \cdot p \geq 0
$$

with the radial component of the momentum

$$
p^{r} \leq 0
$$

Our quantization conditions (5) are that $\phi$ and separately $\pi$ at same $r$ commute whereas according to (价 $\phi-\pi$ commutator is nontrivial in the inward directed light-cone. Thus these can be taken to be implementation of the local stability requirement. 
Minkowski space quantization on hypersurfaces of constant $t$ is covariant under rigid Lorentz transformations of the cartesian $t-\vec{x}$ coordinates. The background geometry of the blackhole in Schwarzschild co-ordinates suggests a preferred slicing for quantiztion as chosen above. The hypersurfaces of constant $r$ are also those with $\operatorname{Tr} K=$ constant where $K$ is the extrinsic curvature of the hypersurface. This choice of foliation is standard in dynamical formulation of gravity [6]. Here the resulting field theory will be covariant under constant translations of the $t$ co-ordinate and rigid rotations centred on the origin already chosen. A quantization scheme based on any other co-ordinates will be inequivalent to the present one.

We now proceed to obtain a representation of this algebra by introducing creation and annihilation operators. Consider the massless scalar wave equation $\square \phi=0$ with $\square$ the appropriate d'Alembertian operator for the black hole interior. Separating this in the $\{t, r, \theta, \phi\}$ coordinates, we obtain the mode functions

$$
h_{\omega l m}(r, t, \Omega) \sim \frac{R_{\omega l}(r)}{r} Y_{l m}(\theta, \phi) e^{-i \omega t}
$$

where $Y_{l m}$ are the spherical harmonics. In the co-ordinate $r^{*}$, the equation satisfied by $R_{\omega l}$ is

$$
\frac{d^{2} R_{\omega l}}{d r^{* 2}}+\left(\omega^{2}-\left[\frac{l(l+1)}{r^{2}}+\frac{2 m}{r^{3}}\right]\left[1-\frac{2 m}{r}\right]\right) R_{\omega l}=0
$$

Writing this in the form

$$
\frac{d^{2} R_{\omega l}}{d r^{* 2}}+k_{\omega}^{2} R_{\omega l}=0
$$

we can identify the positive definite quantity

$$
\begin{aligned}
k_{\omega}^{2} & =\omega^{2}-V^{\mathrm{eff}} \\
& \equiv \omega^{2}-\left[\frac{l(l+1)}{r^{2}}+\frac{2 m}{r^{3}}\right]\left[1-\frac{2 m}{r}\right]
\end{aligned}
$$

with the radial wave-number squared of the mode. The $V^{\text {eff }}$ vanishes at the horizon so that $k_{\omega}=\omega$ there. Further, close to the horizon, i.e., for $2 m-r \ll 2 m, R_{\omega l} \sim$ $e^{ \pm i\left(k_{\omega} r^{*}-\omega t\right)}$. We shall now on take $k_{\omega}$ to be the positive square root of the above equation, and choose $h_{\omega l m}$ to be those mode functions which satisfy near the horizon!

$$
\begin{aligned}
\frac{\partial}{\partial r^{*}} h_{\omega l m}(r, t, \Omega)= & -i k_{\omega} h_{\omega l m}(r, t, \Omega) \\
& (\text { for } \quad r<2 m \text { and } 2 m-r \ll 2 m)
\end{aligned}
$$

\footnotetext{
${ }^{1}$ The $e^{-i \omega t} \psi^{l}(r,-\omega)$ of 佨 correspond to our $h_{\omega l m}^{*}$
} 
i.e., the positive definite eigenvalues are associated with ingoing radial momentum. This is analogous to the condition for positive frequency modes in Minkowski space. With appropriate normalisation, these modes satisfy the completeness relation

$$
\sum_{l m} \int d \omega h_{\omega l m}(r, t, \Omega) h_{\omega l m}\left(r, t^{\prime}, \Omega^{\prime}\right)=\delta_{\Sigma}\left(r, t, \Omega ; r, t^{\prime}, \Omega^{\prime}\right)=\frac{\delta\left(t-t^{\prime}\right) \delta\left(\Omega-\Omega^{\prime}\right)}{r^{2}}
$$

where $\Sigma$ is the surface of quantization, a constant- $r$ hypersurface near the horizon, as before. In the mode expansion we now take summation over modes with the parameter $\omega$ taking both positive and negative values while $k_{\omega}$ remains positive. Accordingly, the interior Fourier expansion near the horizon is

$$
\phi_{i n}=\sum_{l m} \int_{-\infty}^{\infty} \frac{d \omega}{\sqrt{2 \pi} r \sqrt{2 k_{\omega}}}\left(a_{\omega} e^{-i\left(k_{\omega} r^{*}-\omega t\right)} Y_{l m}+b_{\omega}^{\dagger} e^{i\left(k_{\omega} r^{*}-\omega t\right)} Y_{l m}^{*}\right)
$$

where we have written $h_{\omega} \sim e^{-i\left(k_{\omega} r^{*}-\omega t\right)} / r$ as asymptotic forms near the horizon of general modes $h_{\omega l m}$. The indices $l, m$ on $a_{\omega}$ and $b_{\omega}$ have been suppressed. Now imposing the quantization conditions (4), (5) we obtain for the expansion parameters,

$$
\left[a_{\omega}, a_{\omega^{\prime}}^{\dagger}\right]=\delta\left(\omega-\omega^{\prime}\right)=\left[b_{\omega}, b_{\omega^{\prime}}^{\dagger}\right]
$$

and that all other commutators vanish.

We can justify the interpretation of modes implied by conditions (16) by paraphrasing the CPT invariance argument of Weinberg [1]. Consider an observer in the interior who performs an experiment in which a particle is created at point A and is "later", i.e., further down the radial direction is destroyed at B. This radial (temporal in the interior) ordering of events cannot be reversed for any classical events by another observer without a superluminal Lorentz transformation. However for those events separated by spacetime intervals less than the Compton wavelength of the particle, this is not guaranteed. In this case it is possible to observe a particle of the same charge being annihilated at $\mathrm{B}$ before being created at A, thus travelling backward in $r$. The only way causality can be maintained is to insist that a particle of opposite charge travelled forward in $r$, emitted from B and absorbed at A. Eq.s (16) are consistent with this interpretation of the modes.

\section{Recovering the Spectrum condition and CPT}

Next we need to verify that the algebra of the operators corresponding to spacetime symmetries is realised, and in particular that a positive definite operator exists, whose spectrum guarantees the existence of a ground state upon which the spectrum generated

by the creation operators can be built. To begin with we demand the existence of a vacuum or no-particle state $\left|0^{I}\right\rangle$ satisfying

$$
a_{\omega}\left|0^{I}\right\rangle=b_{\omega}\left|0^{I}\right\rangle=0,
$$


The global Killing symmetries of time translation (spacelike in the interior) and rotations on surfaces of constant $r$ are implemented by the operators

$$
\begin{aligned}
K_{t} & =\int d \Lambda T_{t}^{t} \\
K_{\theta} & =\int d \Lambda T_{\theta}^{\theta} \\
K_{\varphi} & =\int d \Lambda T_{\varphi}^{\varphi}
\end{aligned}
$$

with $d \Lambda$ denoting the induced 3-volume on constant $r$ hypersurfaces and with $T$ components obtained in the usual way from the matter Lagrangian. These can be thought of as the infalling mass-energy at any fixed radius and angular momentum content respectively of the quantum field $\phi$. The energy now is not positive definite, but this is the operator corresponding to the energy operator outside. In the vacuum introduced above, we find

$$
\left\langle 0^{I}\left|K_{t}\right| 0^{I}\right\rangle=\left\langle 0^{I}\left|K_{\theta}\right| 0^{I}\right\rangle=\left\langle 0^{I}\left|K_{\varphi}\right| 0^{I}\right\rangle=0
$$

The above vanishing of the expectation values is clear because $\int_{-\infty}^{\infty} d \omega \omega \ldots$ and $\sum_{m=-l}^{l}$ etc. appear. The quantum dynamics is now generated by the operator

$$
K_{r}=\int d \Lambda T_{r}^{r}
$$

The radial momentum density is

$$
\begin{aligned}
& T_{r}^{r}=\Pi_{r} \partial_{r^{*}} \phi+\Pi_{r}^{\dagger} \partial_{r^{*}} \phi^{\dagger}-L \\
& =\frac{1}{\frac{2 m}{r}-1}\left[\partial_{r^{*}} \phi^{\dagger} \partial_{r^{*}} \phi+\dot{\phi}^{\dagger} \dot{\phi}\right]
\end{aligned}
$$

This is clearly positive definite in the black hole interior. Promoted to a quantum operator, $K_{r}$ is thus positive definite. Substituting the mode expansion (15) and using the expression for the volume element on the constant $r$ hypersurface near the horizon

$$
d \Lambda=(2 m / r-1)^{1 / 2} r^{2} \sin \theta d t d \theta d \varphi
$$

we obtain the following expression for normal ordered $K_{r}$

$$
: K_{r}:=r\left(\frac{2 m}{r}-1\right)^{-1 / 2} \int_{-\infty}^{\infty} d \omega k_{\omega}\left(a_{\omega}^{\dagger} a_{\omega}+b_{\omega}^{\dagger} b_{\omega}\right)
$$

This makes the state defined through eq.s (17) a genuine ground state as well as a noparticle state. Note that the sign in front of $k_{\omega}$ in above eqn. is due to our choice the sign of $k_{\omega}>0$ below eqn. (12).

The ground state thus characterised has been shown to be stable 4 ]. One may think of it as the adiabatic vacuum useful to a freely infalling observer. The latter should detect particle production, and the same will be finite if the vacuum specified here is 
used as the template with which to compare his local ground states. This is similar to what happens in Friedmann-Robertson-Walker geometries which have a conformal timelike Killing vector. In the present case we expect copious particle production as the singularity is approached, much as in the collapsing phase of relevant FRW metrics. The normal ordering prescription used may seem arbitrary. But the effect of the infinite contribution discarded manifests itself as higher derivative terms in the effective action for gravity. It is possible to choose a renormalisation prescription such that when one returns to the gauge specified, the numerical values of the renormalised operators will be the same as that obtained by simple normal ordering.

Finally, CPT invariance can be realised by requiring

$$
C \phi C^{\dagger}=\phi^{\dagger}
$$

which implies the effect of $C$ is

$$
a_{\omega} \leftrightarrow b_{\omega}
$$

Similarly, a restricted parity operator corresponding to reflection in the blackhole origin is given by

$$
P^{\Omega} \phi(r, t, \theta, \varphi) P^{\Omega^{\dagger}}=\phi(r, t, \pi-\theta, \varphi+\pi)
$$

which implies, under $P^{\Omega}$

$$
a_{\omega, l, m} \leftrightarrow a_{\omega l,-m}(-1)^{l}
$$

and for vector operators

$$
P^{\Omega} V^{\mu}(r, t, \theta, \varphi) P^{\Omega^{\dagger}}=V_{\mu}(r, t, \pi-\theta, \varphi+\pi)
$$

The $P^{\Omega}$ symmetry is complemented by reversal symmetry of the $t$ co-ordinate

$$
\tilde{T} \phi(r, t, \Omega) \tilde{T}^{\dagger}=\phi(r,-t, \Omega)
$$

such that $\tilde{T}$ is unitary. This gives

$$
a_{\omega} \leftrightarrow a_{-\omega} \text { and } b_{\omega} \leftrightarrow b_{-\omega}
$$

There is no global symmetry reversing the arrow of dynamical evolution since $r$ translations are not a symmetry. However in the adiabatic approximation, a local and antiunitary $r$ reversal operator $\tilde{R}(r)$ may be assumed to exist such that for small increments $\Delta r$,

$$
\tilde{R}(r) \phi(r+\Delta r, t, \Omega) \tilde{R}(r)^{\dagger}=\phi(r-\Delta r, t, \Omega)
$$

\section{The propagator}

Since the inexorable direction of propagation is along the inward radial vector (corresponding to the $t$ variable in the exterior), the propagator in the interior is expected to be $r$-ordered. This is analogous to $t$-ordering in the exterior. 
Such a propagator can be written as

$$
\begin{gathered}
i G\left(x, x^{\prime}\right)=<0^{I}\left|R \phi(x) \phi^{\dagger}\left(x^{\prime}\right)\right| 0^{I}> \\
i G\left(x, x^{\prime}\right)=\theta\left(r^{*}-r^{\prime *}\right)<0^{I}\left|\phi(x) \phi^{\dagger}\left(x^{\prime}\right)\right| 0^{I}>+\theta\left(r^{\prime *}-r^{*}\right)<0^{I}\left|\phi^{\dagger}\left(x^{\prime}\right) \phi(x)\right| 0^{I}>
\end{gathered}
$$

The $\theta$ function defined above is the usual step function. This propagator satisfies the equation

$$
\square_{x} G\left(x, x^{\prime}\right)=\delta\left(x, x^{\prime}\right)=[-g(x)]^{-1 / 2} \delta^{4}\left(x-x^{\prime}\right)
$$

thus making $G\left(x, x^{\prime}\right)$ a Green's function for the wave equation in the Schwarzschild interior geometry.

Near the horizon, the field can be expanded as in eqn.(15). Using the integral representation for the theta function, the expression for $G\left(r^{*}, t ; r^{* \prime}, t^{\prime}\right)$ (suppressing the angular co-ordinates) becomes

$$
G\left(r^{*}, t ; r^{* \prime}, t^{\prime}\right)=\lim _{\epsilon \rightarrow 0+} \int_{-\infty}^{\infty} \frac{d \omega d \Lambda}{(2 \pi)^{2} r r^{\prime}} \frac{e^{-i \Lambda\left(r^{*}-r^{\prime *}\right)+i \omega\left(t-t^{\prime}\right)}}{\Lambda^{2}-k_{\omega}^{2}+i \epsilon}
$$

\section{Conclusion}

We have shown that taking account of the classical inexorably inward motion and incorporating the requirements of $\mathrm{CPT}$, a unique quantization can be obtained for a matter field in the black hole interior. The quantization is of the kind possible in spaces with a conformal Killing vector such as FRW universes. It can be taken to be the QFT set up by the freely infalling observer, the equivalent of a comoving observer in FRW spacetime. We have also shown that imposing microcausality dictates the form of the propagator at least in the co-ordinates used. The quantization is expected to break down close to the classical singularity where semiclassical techniques fail. It would be interesting to ask whether there is any signature of the Hawking radiation detected in this quantization. This requires matching of this QFT to the standard QFT in the exterior.

\section{References}

[1] S. Weinberg Gravitation and Cosmology John Wiley (1973), sec. 2.13.

[2] R. Haag, H. Narnhofer, U. Stein, Comm. Math. Phy. 94, 219 (1984). 
[3] P. Candelas, B. P. Jensen, Phys. Rev. D33, 1596(1986); see also ibid pg. 1590.

[4] D. G. Boulware Phys. Rev. D11, 1404 (1975). Our labelling of mode functions differs from this reference. We treat $r^{*}$ as the natural co-ordinate and make the split according to positive and negative $k_{\omega}$. See our footnote regarding eqn.(13).

[5] C. W. Misner, K. S. Thorne and J. A. Wheeler Gravitation W. H. Freeman (1973), pg. 663

[6] see for example J. Isenberg and J. A. Wheeler in Relativity, Quanta and Cosmology in the Development of the Scientific Thought of Albert Einstein, M. Pantaleo and F. deFinis, ed.s, vol. I, pp 267-293 (1979) 